Pacific Journal of Mathematics

$B$-COMPLETE AND $B_{r}$-COMPLETE TOPOLOGICAL 


\title{
$B$-COMPLETE AND $B_{r}$-COMPLETE TOPOLOGICAL ALGEBRAS
}

\author{
DOMENICO RosA
}

In this note, a topological algebra $A$ is an algebra over the field of complex numbers together with a Hausdorff locally convex topology which makes multiplication jointly continuous. $A$ is called $B$-complete $\left(B_{r}\right.$-complete) if every continuous (continuous, one-to-one) and almost open algebra homomorphism from $A$ onto any topological algebra is open. For a completely regular space $X, C(X)$ is the algebra of all continuous complex-valued functions with the usual pointwise operations and the compact-open topology. The main theorem states that $C(X)$ is a $B$-complete algebra iff $X$ is a $k$-space.

Recall that a linear map between two locally convex spaces is called almost open if the closure of the image of every neighborhood of zero is a neighborhood of zero. Pták [7] called a locally convex space $E B$ complete if every continuous and almost open linear map from $E$ onto any locally convex space is open. A general treatment of $B$-complete spaces may be found in [2]. Our study of $B$-complete algebras is motivated by a problem raised in [7]; namely, what conditions on $X$ are necessary and sufficient for $C(X)$ to be a $B$-complete space. Necessary conditions are that $X$ be a normal $k$-space, a result which is implicit in [7]. W. H. Summers [11] has obtained some partial results for weighted spaces of bounded continuous functions.

We shall investigate this problem within the framework of topological algebras rather than locally convex spaces. The definition of a $B$-complete algebra is motivated by T. Husain's extension of the notion of $B$-completeness for suitable classes of locally convex spaces [2] and for topological groups [3]. In Section 2 we obtain a characterization of a $B$-complete algebra $A$ in terms of its topological dual $A^{\prime}$. In Section 3 we prove that $C(X)$ is a $B$-complete algebra iff $X$ is a $k$-space. In Section 4 several counterexamples are provided, and in Section 5 a closed graph theorem is stated.

A convex subset $U$ of an algebra $A$ is called $m$-convex if $U \cdot U \subset$ $U$. A topological algebra $A$ is called locally $m$-convex (LMC) if there exists a basis for the neighborhoods of zero consisting of closed $\mathrm{m}$ convex and circled sets. Equivalently, $A$ is LMC iff its topology is generated by a set $\left\{p_{i} ; i \in I\right\}$ of sub-multiplicative seminorms $\left(p_{i}(x y) \leqq p_{i}(x) p_{i}(y)\right.$ for $\left.i \in I, x, y \in A\right)$. Clearly $C(X)$ is LMC; simply take the seminorms $\left\{p_{K}: K\right.$ is a compact subset of $\left.X\right\}$ defined by 
$p_{K}(f)=\sup \{|f(x)|: x \in K\}$, for $f \in C(X)$. For $b>0$, we will use the notation $N(K, b)=N\left(p_{K}, b\right)=\left\{f \in C(X):\left|p_{K}(f)\right| \leqq b\right\}$ to denote basic closed neighborhoods of zero in $C(X)$.

2. A characterization of $B$-complete algebras. It follows directly from the definitions that every $B$-complete algebra is $B_{r}$-complete, and that the quotient of a $B$-complete algebra modulo a closed ideal is $B$-complete. Also, every topological algebra which is a $B$-complete space is a $B$-complete algebra, in particular, Banach algebras and complete metrizable topological algebras are $B$-complete algebras. For later reference we now state the following two lemmas whose proofs are immediate. At times subscripts will be used to denote topologies.

Lemma 2.1. Let $A_{u}$ be a topological algebra. Then the following statements are equivalent.

(a) $A_{u}$ is a $B_{r}$-complete algebra.

(b) Whenever the identity map $A_{u} \rightarrow A_{v}, A_{v}$ a topological algebra, is continuous and almost open, it follows that $u=v$.

LEMMA 2.2. A topological algebra $A$ is a $B$-complete algebra iff $A / I$ is a $B_{r}$-complete algebra for every closed ideal $I$ of $A$.

Let $A$ be a topological algebra. A linear subspace $S$ of $A^{\prime}$ is called almost closed if $S \cap U^{0}$ is $w^{*}$-closed for every neighborhood $U$ of zero in $A$, where $U^{0}$ is the polar of $U . S$ is said to be an $m$-subspace if whenever $h \in S$ then $h \circ l_{a}$ and $h \circ r_{a}$ are in $S$, where $l_{a}, r_{a}: A \rightarrow A$ are left and right multiplication by $a$, respectively. It can be readily checked that the polar of an ideal is an $m$-subspace and the polar of an $m$-subspace is an ideal.

The following theorem extends theorem IV, 8.1 of [9] to algebras. The author would like to thank Seth Warner for suggesting it.

TheOREM 2.3. Let $A$ be a topological algebra.

(a) $A$ is a $B_{r}$-complete algebra iff every $w^{*}$-dense and almost closed $m$-subspace of $A^{\prime}$ coincides with $A^{\prime}$.

(b) $A$ is a $B$-complete algebra iff every almost closed $m$-subspace of $A^{\prime}$ is $w^{*}$-closed.

Proof. (a) Suppose $A$ is a $B_{r}$-complete algebra. Let $S$ be a dense and almost closed $m$-subspace of $A^{\prime}$, and let $u$ be the Hausdorff locally convex topology on $A$ defined by the family $\left\{\left(U^{0} \cap S\right)^{0}\right\}$ where $U$ varies over all neighborhoods of zero in $A$. As in [2, Theorem 5, p. 54], the identity map id: $A \rightarrow A_{u}$ is continuous and almost open, and $\left(A_{u}\right)^{\prime}=$ $S$. It remains to show that $A_{u}$ is a topological algebra. Since $S$ is an 
$m$-subspace it follows that $l_{a}, r_{a}: A_{v} \rightarrow A_{v}$ are continuous for every $a \in A$, where $v$ is the weak topology determined by $S$. Using the additional facts that id: $A \rightarrow A_{u}$ is almost open and that the convex circled sets which are $u$-closed are also $v$-closed, we can easily conclude that $l_{a}, r_{a}: A_{u} \rightarrow A_{u}$ are continuous for every $a \in A$. To show that multiplication is jointly continuous, let $V$ be a closed neighborhood of zero in $A_{u}$. Then there exists a neighborhood $U$ of zero in $A$ such that $U \cdot U \subset V$. Since multiplication is separately continuous in $A_{u}$, it follows as in [4, Lemma 1.4(b)] that $\mathrm{cl}_{u}(U) \cdot \mathrm{cl}_{u}(U) \subset V$. Since the identity map is almost open, the factors on the left are $u$-neighborhoods of zero, hence $A_{u}$ is a topological algebra. By our hypothesis id: $A \rightarrow A_{u}$ is open, consequently $S=\left(A_{u}\right)^{\prime}=A^{\prime}$.

In view of Lemma 2.1 , for the converse we may suppose that id: $A \rightarrow A_{u}, A_{u}$ a topological algebra, is continuous and almost open. By [2, Proposition 14, p. 54], $\left(A_{u}\right)^{\prime}$ is an almost closed subspace of $A^{\prime}$, and clearly it is also a $w^{*}$-dense subspace. Since multiplication is jointly continuous in $A_{u}$, it follows that $\left(A_{u}\right)^{\prime}$ is an $m$-subspace of $A^{\prime}$. By hypothesis $\left(A_{u}\right)^{\prime}=A^{\prime}$ which implies that $A$ and $A_{u}$ have the same closed, convex and circled sets. This implies that id: $A \rightarrow A_{u}$ is actually open, hence $A$ is a $B_{r}$-complete algebra by Lemma 2.1 .

(b) Suppose $A$ is a $B$-complete algebra. Let $S$ be an almost closed $m$-subspace of $A^{\prime}$. Then $A / S^{0}$ is a $B$-complete algebra, and $S$ is a $w^{*}$-dense and almost closed $m$-subspace of $\left(A / S^{0}\right)^{\prime}$. By (a) $S=$ $\left(A / S^{0}\right)^{\prime}=S^{00}$ is $w^{*}$-closed in $A^{\prime}$.

In view of Lemma 2.2, to prove the converse it suffices to show that $A / I$ is $B_{r}$-complete for every closed ideal $I$ of $A$. So let $S$ be a $w^{*}$-dense and almost closed $m$-subspace of $(A / I)^{\prime}$, and let $q: A \rightarrow A / I$ be the quotient map. As in [2, Theorem 1, p. 46] $q^{\prime}(S)$ is an almost closed subspace of $A^{\prime}$, where $q^{\prime}$ is the transpose of $q$. Since $h \circ q \circ r_{a}=$ $\left(h \circ r_{q(a)}\right) \circ q$ and $h \circ q \circ l_{a}=\left(h \circ l_{q(a)}\right) \circ q$ for every $a \in A$, it follows that $q^{\prime}(S)$ is also an $m$-subspace of $A^{\prime}$. By hypothesis $q^{\prime}(S)$ is closed. Thus $S \cong q^{\prime}(S)=I^{0} \cong(A / I)^{\prime}$ and consequently $A / I$ is a $B_{r}$-complete algebra. By Lemma $2.2, A$ is a $B$-complete algebra.

Sulley [10] proved a very useful criterion for determining when dense subgroups of $B_{r}$-complete and $B$-complete topological abelian groups inherit the respective properties. The same criterion can be adapted for topological algebras. The proof is essentially the same and is therefore omitted. It should be pointed out that the proof of Theorem 1 in [10] uses the existence of completions. The same proof holds for topological algebras since the completion (of the additive group) of a topological algebra is again a topological algebra.

THEOREM 2.4. Let $B$ be a dense subalgebra of a topological algebra A. 
(a) $B$ is a $B_{r}$-complete algebra iff $A$ is a $B_{r}$-complete algebra and $B$ has nonzero intersection with every nonzero closed ideal of $A$.

(b) $B$ is a $B$-complete algebra iff $A$ is a $B$-complete algebra and $B \cap I$ is dense in I for every closed ideal I of $A$.

CORAllaRy 2.5. Let $C^{*}(X)$ be the subalgebra of $C(X)$ consisting of all bounded functions.

(a) $C^{*}(X)$ is a $B_{r}$-complete algebra iff $C(X)$ is such.

(b) $C^{*}(X)$ is a $B$-complete algebra iff $C(X)$ is such.

Proof. Since $C^{*}(X)$ is a dense subalgebra of $C(X)$, (a) and (b) will follow if we show that $I \cap C^{*}(X)$ is dense in $I$ for every closed nonzero ideal $I$ of $C(X)$. So let $I$ be a closed nonzero ideal of $C(X)$. By [6, Theorem 2.1] $I=I_{F}=\{f \in C(X): f(F)=\{0\}\}$ for some proper closed $F \subset X$. Let $f \in I_{F}$ and let $N(K, f, b)$ be a basic neighborhood of $f$. Let $r=\sup \{|f(x)|: x \in K\}$ and let $G=\{x \in X:|f(x)| \geqq r+1\}$. Then $G$ is a closed subset of $X$ and $G \cap K=\varnothing$. Since $X$ is completely regular, there exists $g \in C^{*}(X), 0 \leqq g \leqq 1$, such that $g(K)=\{1\}$ and $g(G)=$ $\{0\}$. Consequently, $g f \in C^{*}(X) \cap N(K, f, b)$; hence, $I \cap C^{*}(X)$ is dense in $I$. The corollary follows from the theorem.

COROllary 2.6. The subalgebra $P$ of $C([0,1])$ consisting of all polynomials is not a $B_{r}$-complete algebra.

Proof. Since $C([0,1])$ is a Banach algebra it is a $B_{r}$-complete algebra. Let $I$ be the closed nonzero ideal of all functions which are zero on $[0,1 / 2]$. Since $P$ is dense in $C([0,1])$ and since $I \cap P=\{0\}$ it follows by Theorem $2.4(\mathrm{a})$ that $P$ is not a $B_{r}$-complete algebra.

As a consequence of Corollary 2.5 it follows that a $B$-complete algebra need not be complete. Let $R$ be the real line with the usual topology. Then $C(R)$ is complete and metrizable, hence a $B$-complete algebra. Thus $C^{*}(R)$ is a $B$-complete algebra which is not complete. Corollary 2.6 shows that a normed algebra need not be $B_{r}$-complete.

3. Necessary and sufficient conditions for $C(X)$ to be a $B$-complete algebra. Recall that a subset $S$ of a topological space $X$ is $k$-closed if $S$ has closed intersection with every compact subset of $X$, and $X$ is called a $k$-space if every $k$-closed subset of $X$ is closed. (For example, locally compact spaces and first countable spaces are $k$-spaces.) 
THEOREM 3.1. $C(X)$ is a $B_{r}$-complete algebra iff every dense and $k$-closed subset of $X$ coincides with $X$.

Proof. That the above condition is necessary was essentially proved by Pták [7, Theorem 6.4]. For suppose that $C(X)$ is a $B_{r}$-complete algebra, and let $S$ be a dense and $k$-closed subset of $X$. Let $u$ be the topology of uniform convergence on the compact subsets of $S$. Clearly $C(X)_{u}$ is LMC, hence a topological algebra. By [7, Theorem 6.4] the identity map $C(X) \rightarrow C(X)_{u}$ is continuous and almost open, hence open by our hypothesis. It follows directly that $S=X$.

In view of Lemma 2.1 , to prove the converse we may suppose that the identity map id: $C(X) \rightarrow C(X)_{u}$ is continuous and almost open, where $C(X)_{u}$ is a topological algebra. Let $S=\left\{x \in X: h_{x}\right.$ is $u$ continuous $\}$, where $h_{x}$ is defined by $h_{x}(f)=f(x)$ for $f \in C(X)$. We will show that $S$ is a dense and $k$-closed subset of $X$.

The first step is to show that the topology $u$ is generated by a subset of the seminorms $\left\{p_{K}: K\right.$ is a compact subset of $\left.X\right\}$. Since $C(X)_{u}$ is a topological algebra, by [4, Lemma 1.4(b)] it follows that $\operatorname{cl}_{u} N(K, b)$ is $m$-convex for every compact $K \subset X$ and every $0 \leqq b \leqq 1$. Since the identity map is almost open, these sets form a basis for the topology $u$; hence $C(X)_{u}$ is LMC and $u$ is generated by a set of submultiplicative seminorms $\left\{p_{j}\right\}, j \in J$. Let $p \in\left\{p_{j}\right\}, j \in J$. Since $p$ is submultiplicative, $\operatorname{ker}(p)$ is a closed ideal of $C(X)_{u}$, hence also closed in $C(X)$. By [6, Theorem 2.1] $\operatorname{ker}(p)=I_{H}$ for some closed $H \subset X$. Since $p$ is continuous on $C(X), N(K, b) \subset N(p, 1)$ for some compact subset $K$ of $X$ and some $b>0$. (Note that $N(K, b)$ need not be a $u$-neighborhood of zero.) This implies $I_{K}=\operatorname{ker}\left(p_{K}\right) \subset \operatorname{ker}(p)=I_{H}$, which in turn implies that $H \subset K$. Thus $H$ is a compact subset of $X$. Let $h: C(X) \rightarrow C(X) / I_{H}=C(H)$ and $k: C(X)_{u} \rightarrow C(X)_{u} / I_{H}=C(H)_{u}$ be the respective quotient homomorphisms, and let id ${ }^{*}$ be the unique oneto-one and onto homomorphism such that $i d^{*} \circ h=k \circ i d$. Since id is continuous and almost open, so is $i d^{*}$. Since $I_{H}$ is $u$-closed, $C(H)_{u}$ is a (Hausdorff) topological algebra, hence $i d^{*}$ is open since $C(H)$ is a Banach algebra. It follows that the seminorm $p_{H}$ is continuous on $C(X)_{u}$ and that $N\left(p_{H}, b\right) \subset N(p, 1)$ for some $b>0$. Since $p$ was chosen arbitrarily we can conclude that the topology $u$ is generated by a subset of $\left\{p_{K}: K\right.$ is a compact subset of $\left.X\right\}$.

A simple argument shows that:

$S=\cup\left\{H: H \subset X\right.$ is compact and $p_{H}$ is continuous on $\left.C(X)_{u}\right\}$.

(1) $=\cup\left\{H: H \subset X\right.$ is compact and $N(H, 1)$ is a nbd. of zero in $\left.C(X)_{u}\right\}$.

Since $X$ is completely regular and since $u$ is a Hausdorff topology 
generated by seminorms of the form $p_{H}$, it follows directly that $S$ is a dense subset of $X$. To show that $X$ is $k$-closed, let $K$ be a compact subset of $X$. Since the identity map is almost open, $\operatorname{cl}_{u} N(K, 1)$ is a neighborhood of zero in $C(X)_{u}$. Since $N(K \cap S, 1)$ is closed in $C(X)_{u}$, it follows that $-\operatorname{cl}_{u} N(K, 1) \subset N(K \cap S, 1)=N(\operatorname{cl}(K \cap S), 1)$ and consequently the latter is also a neighborhood of zero in $C(X)_{u}$. By (1) it follows that $\operatorname{cl}(K \cap S) \subset S$. Thus $\operatorname{cl}(K \cap S) \subset K \cap S$, which implies that $S$ is $k$-closed. By our hypothesis $S=X$, hence $h_{x}$ is $u$-continuous for each $x \in X$. Consequently, $N(K, 1)$ is $u$-closed for each compact $K \subset$ $X$, hence a $u$-neighborhood of zero since id: $C(X) \rightarrow C(X)_{u}$ is almost open. Thus $C(X)=C(X)_{u}$ and in view of Lemma 2.1 it follows that $C(X)$ is a $B_{r}$-complete algebra.

From the above theorem it follows that $C(X)$ is a $B_{r}$-complete algebra whenever $X$ is a $k$-space. Example 4.2 shows that the converse is false.

For a closed subset $F$ of $X, C_{F}(X)$ is the topological subalgebra of $C(F)$ consisting of all functions which are restrictions of members of $C(X)$. Clearly, $C_{F}(X)$ is a dense subalgebra of $C(F)$ and the restriction map $r: C(X) \rightarrow C_{F}(X)$ is continuous. Pták [7, Theorem 6.7] showed that $r$ is open, hence $C_{F}(X) \cong C(X) / I_{F}$, the latter having the quotient topology from $C(X)$.

TheOREM 3.2. $C(X)$ is a $B$-complete algebra iff $X$ is a $k$-space.

Proof. Suppose $C(X)$ is a $B$-complete algebra. Let $S$ be a $k$-closed subset of $X$ and let $F=\operatorname{cl}(S)$. Then $S$ is a dense and $k$-closed subset of $F$. Since $C_{F}(X) \cong C(X) / I_{F}$, it follows that $C_{F}(X)$ is also a $B$-complete algebra. Since $C_{F}(X)$ is dense in $C(F)$, by Theorem 2.4(b) it follows that $C(F)$ is also a $B$-complete algebra. By Theorem 3.1, $S=F$ implying that $X$ is a $k$-space.

Conversely, suppose that $X$ is a $k$-space. In view of Lemma 2.2, to show that $C(X)$ is a $B$-complete algebra, it suffices to show that the quotient of $C(X)$ modulo any closed ideal is a $B_{r}$-complete algebra. So let $I$ be a closed ideal of $C(X)$. By [6, Theorem 2.1] $I=I_{F}$ for some closed $F \subset X$. Since $k$-spaces are closed hereditary, $F$ is also a $k$-space, hence by Theorem $3.1 C(F)$ is a $B_{r}$-complete algebra. Since $C_{F}(X)$ is dense in $C(X)$ and has nonzero intersection with every nonzero closed ideal of $C(F)$, it follows from Theorem 2.4(a) that $C_{F}(X)$ is also a $B_{r}$-complete algebra. Thus $C_{F}(X) \cong C(X) / I_{F}=C(X) / I$ is a $B_{r}$ complete algebra. By Lemma 2.2 it follows that $C(X)$ is a $B$-complete algebra.

Corollary 3.3. If $C(X)$ is a $B$-complete space, then $X$ is a normal $k$-space. 
Proof. That $X$ is a $k$-space follows from Theorem 3.2. To show that $X$ is normal, let $F$ be a closed subset of $X$. Since the restriction map $r: C(X) \rightarrow C_{F}(X)$ is continuous and open it follows that $C_{F}(X)$ is also a $B$-complete space. Since $B$-complete spaces are complete $[9,8.1$ p. 162], $C_{F}(X)=C(F)$. By the Urysohn-Tychonoff theorem $X$ is normal.

Theorem 3.2 gives rise to a large class of $B$-complete algebras which are not $B$-complete spaces. If $X$ is a $k$-space which is not normal, in particular the Tychonoff plank, then $C(X)$ is a $B$-complete algebra but not a $B$-complete space.

4. Counterexamples. In this section we will provide examples to show that a complete algebra need not be $B_{r}$-complete and that a $B_{r}$-complete algebra need not be $B$-complete.

Let $R$ and $R^{2}$ be the real line and the plane with their usual topologies. If $X \subset R^{2}$ and $x \in X$, then a function $f: X \rightarrow R$ is called separately continuous at $x$ provided $f \mid(L \cap X)$ is continuous at $\dot{x}$ if $L$ is either the horizontal or the vertical line through $x$ in $R^{2}$. A completely regular space $X$ is called a $k_{R}$-space is every $f: X \rightarrow R$, whose restriction to every compact $K \subset X$ is continuous, is continuous on $X$. It is well known that $C(X)$ is complete iff $X$ is a $k_{R}$-space. Every completely regular $k$-space is a $k_{R}$-space. That the converse is false was first shown by an example of M. Katětov which appeared in [7, Theorem 6.17].

EXAmPLE 4.1. Let $X_{u}$ be $R^{2}$ with the usual topology and let $F$ be the set of all $f: X_{u} \rightarrow R$ which are separately continuous. Let $v$ be the coarsest topology on $X$ making every $f \in F$ continuous. Clearly $X_{v}$ is completely regular. It was shown in [7, Theorem 6.16] that $X_{v}$ is a $k_{R}$-space which has a proper dense and $k$-closed subset. By Theorem 3.1 $C\left(X_{v}\right)$ is not a $B_{r}$-complete algebra; however, it is complete since $X$ is a $k_{R}$-space.

EXAMPLE 4.2. Let $N$ be the natural numbers and let $p \in \beta N \backslash N$, where $\beta N$ is the Stone-Čech compactification of $N$, and let $X=$ $\beta N \amalg(N \cup\{p\})$ be the disjoint topological union. Let $Y$ be the quotient of $X$ which is obtained by identifying the element $p \in \beta N$ with the element $p \in N \cup\{p\}$, and let $f: X \rightarrow Y$ be the quotient map. It can be checked easily that $Y$ is completely regular and that $N \cup\{p\} \cong$ $f(N \cup\{p\})$. Now $f(N)$ (the subset of $f(N \cup\{p\})$ ) is not closed, however, from the counterexample following Proposition 2.2 of [1] it follows that $f(N) \cap K$ is finite for every compact $K \subset Y$. Thus $f(N)$ is $k$-closed but not closed, hence $Y$ is not a $k$-space. Let $D$ be a dense and $k$-closed subset of $Y$. Since $f(N)$ is a discrete subset of both $f(N \cup\{p\})$ and $f(\beta N)$, it follows that $f(N) \subset f(N \cup\{p\}) \cap D$ and $f(N) \subset f(\beta N) \cap$ 
$D$. Since $D$ is $k$-closed $f(\beta N)=f(\beta N) \cap D$, and thus $D=Y$. By Theorem $3.1 C(Y)$ is a $B_{r}$-complete algebra, but $C(Y)$ is not a $B$-complete algebra since $Y$ is not a $k$-space. It should be pointed out that the completion of $C(Y)$ is isomorphic to $C(\beta N \amalg N)$ which is a $B$-complete algebra.

EXAMPLE 4.3. To show that a complete and $B_{r}$-complete algebra need not be $B$-complete, we will use the following topological space constructed in [5, Section 3]. Let $X_{u}$ be as in Example 4.1, and let $A$ be the $x$-axis. Let $F$ be the set of all $f: X_{u} \rightarrow R$ which are continuous on $X_{u} \backslash A$ and separately continuous at every $x \in A$, and let $w$ be the coarsest topology making every $f \in F$ continuous. As in [5, Section 3], $X_{w}$ is a $k_{R}$-space which is not a $k$-space; also, $u=w$ on $X \backslash A$ and on every vertical and every horizontal line.

Let $D$ be a dense and $k$-closed subset of $X_{w}$. Since $X_{w} \backslash A$ is open, $D \cap\left(X_{w} \backslash A\right)$ is dense and $k$-closed in $X_{w} \backslash A$; hence, $D \cap\left(X_{w} \backslash A\right)=$ $X_{w} \backslash A$ since the latter is a $k$-space. For $(a, 0) \in A$, let $B_{a}=$ $\{(a, b):-1 \leqq b \leqq 1\}$. Since $B_{a}$ is $w$-compact, $D$ is $k$-closed, and $X_{w} \backslash A \subset D$, it follows that $B_{a} \cap D=B_{a}$. Thus $A \subset D$ and so $D=$ $X_{w}$. So $C\left(X_{w}\right)$ is a complete and $B_{r}$-complete algebra, but not a $B$-complete algebra since $X_{w}$ is not a $k$-space.

The topological spaces of Examples 4.1 and 4.2 can be used to show that a closed subalgebra of a $B$-complete algebra need not be $B_{r}$ complete, and that the quotient of a $B_{r}$-complete algebra need not be $B_{r}$-complete.

EXAMPLE 4.4. Let $X_{v}$ be as in Example 4.1 and let $Y=\amalg K$ be the disjoint topological union of all compact $K \subset X_{v}$. Let $f: Y \rightarrow X_{v}$ be the map which embeds each $K$ identically into $X_{v}$. Since every compact subset of $X_{v}$ is the image of some compact subset of $Y$, it follows that the induced homomorphism $C(f): C\left(X_{v}\right) \rightarrow C(Y)$ is a topological embedding. Since $C\left(X_{v}\right)$ is complete it is homeomorphic to a closed subalgebra of $C(Y)$. Since $Y$ is locally compact $C(Y)$ is $B$-complete, however, $C\left(X_{v}\right)$ is not even a $B_{r}$-complete algebra.

Example 4.5. Let $X, Y$ and $f: X \rightarrow Y$ be as in Example 4.2. Consider the closed subset $S=f(N \cup\{p\})$, and let $r: C(Y) \rightarrow C(S)$ be the restriction map. Since $Y$ is regular and $\sigma$-compact it is normal, hence $r$ is onto. By [7, Theorem 6.7] $r$ is continuous and open. Since $f(N)$ is a proper dense and $k$-closed subset of $S, C(S)$ is not a $B_{r}$-complete algebra, although $C(Y)$ is $B_{r}$-complete.

We conclude this section by pointing out that $B$-complete algebras are not productive. This follows from the fact that $B$-complete spaces 
are not productive [2, (1) page 48], and the fact that every $B$-complete space is a $B$-complete algebra if we take multiplication to be zero $(x y=0$ for all $x, y)$.

\section{A closed graph theorem for $B_{r}$-complete} algebras. A closed graph theorem can be obtained for $B_{r}$-complete algebras analogous to the one proved by $T$. Husain [3, pp. 95-96] for $B$-complete groups. Except for a few minor points, the proof is essentially the same and so only a sketch is given. Recall that a linear map $f: E \rightarrow F$ is called almost continuous if $\mathrm{cl}\left(f^{-1}(V)\right)$ is a neighborhood of zero whenever $V$ is a neighborhood of zero, and $f$ is said to have a closed graph if $\{(x, f(x)): x \in E\}$ is a closed subset of $E \times F$.

Theorem 5.1. Let $B$ be a $B_{r}$-complete algebra and let $A$ be $a$ topological algebra. Let $h: A \rightarrow B$ be an almost continuous homomorphism having a closed graph and such that $h(A)$ is dense in $B$. Then $h$ is continuous.

Proof. Let $u$ be the initial topology of $B$ and let $v$ be the topology generated by the sets

$$
U^{*}=\operatorname{cl}\left(h\left(\operatorname{cl}\left(h^{-1}(U)\right)\right)\right)
$$

where $U$ varies over all neighborhoods of zero in $B_{u}$. Since $h(A)$ is dense in $B_{u}$ each $U^{*}$ is a neighborhood of zero in $B_{u}$, hence the identity map id: $B_{u} \rightarrow B_{v}$ is continuous. Since the graph of $h$ is closed, it follows as in [3] that $v$ is a Hausdorff topology. Clearly $v$ is a locally convex topology, and since $V^{2} \subset U$ implies that $\left(V^{*}\right)^{2} \subset U^{*}$ it follows that $B_{v}$ is a topological algebra. Since $h$ is almost continuous, $h \circ i d$ is continuous and consequently $U^{*} \subset \mathrm{cl}_{v}(U)$, implying that $i d: B_{u} \rightarrow B_{v}$ is almost open. Since $B_{u}$ is a $B_{r}$-complete algebra, the identity map is open and consequently $h$ is continuous.

In closing we note that, in general, the assumption that $h(A)$ is dense in $B$ cannot be dropped. This is an immediate consequence of the fact that $B_{r}$-complete algebras are not closed hereditary.

Let $B$ be a $B_{r}$-complete algebra having a closed subalgebra $A$ which is not $B_{r}$-complete (e.g., Ex. 4.4). Then there exists another topology $u$ on $A$ such that $A_{u}$ is a topological algebra and the identity map $A \rightarrow A_{u}$ is continuous and almost open, but not open. It follows that the embedding $A_{u} \rightarrow B$ is almost continuous and has a closed graph, but is not continuous.

Portions of this paper are drawn from a chapter in the author's $\mathrm{Ph}$. D. thesis, written at McMaster University under the direction of Dr. Taqdir Husain. The author gratefully acknowledges the financial assistance of McMaster University. 


\section{REFERENCES}

1. H. Buchwalter and J. Schmets, Sur quelque propriétés de l'espace $C_{s}(T)$, J. Math. Pure et Appl., 52 (1973).

2. T. Husain, The open mapping and closed graph theorems in topological vector spaces, Oxford Mathematical Monographs, Oxford, 1965.

3. - Introduction to topological groups, Saunders, Philadelphia, 1966.

4. E. Michael, Locally multiplicatively-convex topological algebras, Mem. Amer. Math. Soc., 11 (1952).

5. —, On $k$-spaces, $k_{R}$-spaces, and $k(X)$, Pacific J. Math., 47 (1973).

6. P. D. Morris and D. E. Wulbert, Functional representation of topological algebras, Pacific J. Máth., 22 (1967), 323-337.

7. V. Pták, On complete topological linear spaces, (Russian, English summary), Czechoslovak Math. J., 3 (78), (1953), 301-364.

8. Completeness and the open mapping theorem, Bull. Soc. Math. France, 86 (1958), 41-74.

9. H.-H. Schaefer, Topological vector spaces, Springer-Verlag, New York, 1971.

10. L. J. Sulley, A note on B-and B,-complete topological Abelian groups, Pro. Camb. Phil. Soc., 66 (1967), 275-279.

11. W. H. Summers, Full-completeness in weighted spaces, Canad. J. Math., 22 (1970), 1196-1207.

Received February 6, 1975.

16 Niles St., APt. 311

HARTFORD, CT. 06105 


\section{PACIFIC JOURNAL OF MATHEMATICS}

\section{EDITORS}

RICHARD ARENS (Managing Editor)

University of California

Los Angeles, California 90024

\author{
R. A. Beaumont \\ University of Washington \\ Seattle, Washington 98105
}

\section{J. DugundII}

Department of Mathematics

University of Southern California

Los Angeles, California 90007

D. Gilbarg and J. Milgram

Stanford University

Stanford, California 94305

\section{ASSOCIATE EDITORS}
E. F. BECKENBACH
B. H. NeumanN
F. WoLF
K. YoshidA

\section{SUPPORTING INSTITUTIONS}

\author{
UNIVERSITY OF BRITISH COLUMBIA \\ CALIFORNIA INSTITUTE OF TECHNOLOGY \\ UNIVERSITY OF CALIFORNIA \\ MONTANA STATE UNIVERSITY \\ UNIVERSITY OF NEVADA \\ NEW MEXICO STATE UNIVERSITY \\ OREGON STATE UNIVERSITY \\ UNIVERSITY OF OREGON \\ OSAKA UNIVERSITY
}

\author{
UNIVERSITY OF SOUTHERN CALIFORNIA \\ STANFORD UNIVERSITY \\ UNIVERSITY OF TOKYO \\ UNIVERSITY OF UTAH \\ WASHINGTON STATE UNIVERSITY \\ UNIVERSITY OF WASHINGTON \\ AMERICAN MATHEMATICAL SOCIETY
}

The Supporting Institutions listed above contribute to the cost of publication of this Journal, but they are not owners or publishers and have no responsibility for its contents or policies.

Mathematical papers intended for publication in the Pacific Journal of Mathematics should be in typed form or offset-reproduced (not dittoed), double spaced with large margins. Underline Greek letters in red, German in green, and script in blue. The first paragraph or two must be capable of being used separately as a synopsis of the entire paper. Items of the bibliography should not be cited there unless absolutely necessary, in which case they must be identified by author and Journal, rather than by item number. Manuscripts, in duplicate, may be sent to any one of the four editors. Please classify according to the scheme of Math. Reviews, Index to Vol. 39. All other communications should be addressed to the managing editor, or Elaine Barth, University of California, Los Angeles, California, 90024.

100 reprints are provided free for each article, only if page charges have been substantially paid. Additional copies may be obtained at cost in multiples of 50.

The Pacific Journal of Mathematics is issued monthly as of January 1966. Regular subscription rate: $\$ 72.00$ a year (6 Vols., 12 issues). Special rate: $\$ 36.00$ a year to individual members of supporting institutions.

Subscriptions, orders for back numbers, and changes of address should be sent to Pacific Journal of Mathematics, 103 Highland Boulevard, Berkeley, California, 94708.

PUBLISHED BY PACIFIC JOURNAL OF MATHEMATICS, A NON-PROFIT CORPORATION Printed at Jerusalem Academic Press, POB 2390, Jerusalem, Israel.

\section{Copyright (C) 1975 Pacific Journal of Mathematics All Rights Reserved}




\section{Pacific Journal of Mathematics

Vol. 60, No. $2 \quad$ October, 1975

Waleed A. Al-Salam and A. Verma, A fractional Leibniz q-formula ........... 1

Robert A. Bekes, Algebraically irreducible representations of $L_{1}(G) \ldots \ldots \ldots \ldots 11$

Thomas Theodore Bowman, Construction functors for topological

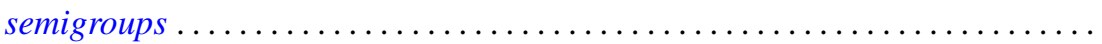

Stephen LaVern Campbell, Operator-valued inner functions analytic on the

closed disc. II .........................................

Leonard Eliezer Dor and Edward Wilfred Odell, Jr., Monotone bases in $L_{p} \ldots \ldots$.

Yukiyoshi Ebihara, Mitsuhiro Nakao and Tokumori Nanbu, On the existence of

global classical solution of initial-boundary value problem for

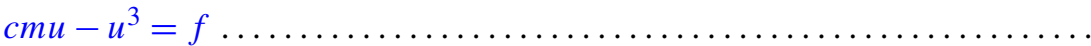

Y. Gordon, Unconditional Schauder decompositions of normed ideals of

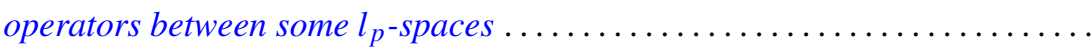

Gary Grefsrud, Oscillatory properties of solutions of certain nth order functional

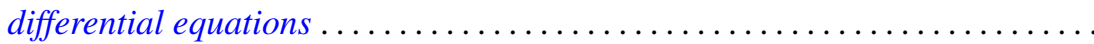

Irvin Roy Hentzel, Generalized right alternative rings ...................

Zensiro Goseki and Thomas Benny Rushing, Embeddings of shape classes of compacta in the trivial range .................................

Emil Grosswald, Brownian motion and sets of multiplicity . .

Donald LaTorre, A construction of the idempotent-separating congruences on a

bisimple orthodox semigroup .

Pjek-Hwee Lee, On subrings of rings with involution ...

Marvin David Marcus and H. Minc, On two theorems of Frobenius ...

Michael Douglas Miller, On the lattice of normal subgroups of a direct

product. .

Grattan Patrick Murphy, A metric basis characterization of Euclidean space

Roy Martin Rakestraw, A representation theorem for real convex functions ....

Louis Jackson Ratliff, Jr., On Rees localities and $H_{i}$-local rings ...

Simeon Reich, Fixed point iterations of nonexpansive mapping . .

Domenico Rosa, $B$-complete and $B_{r}$-complete topological algebras ...

Walter Roth, Uniform approximation by elements of a cone of real-valued

functions ....

Helmut R. Salzmann, Homogene kompakte projektive Ebenen

Jerrold Norman Siegel, On a space between $B H$ and $B_{\infty} \ldots$

235

Robert C. Sine, On local uniform mean convergence for Markov operators

James D. Stafney, Set approximation by lemniscates and the spectrum of an

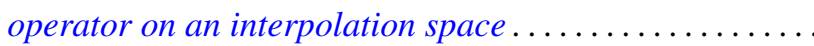

Árpád Száz, Convolution multipliers and distributions .......

Kalathoor Varadarajan, Span and stably trivial bundles ..........

Robert Breckenridge Warfield, Jr., Countably generated modules over

commutative Artinian rings....................... 